\title{
Correlation between superoxide dismutase 1 and 2 polymorphisms and susceptibility to oral squamous cell carcinoma
}

\author{
YING LIU $^{1,2}$, LAGABAIYILA ZHA ${ }^{3}$, BO LI ${ }^{1,2}$, LOUQIANG ZHANG $^{1,2}$, TAO YU ${ }^{4}$ and LONGJIANG LI ${ }^{1,2}$ \\ ${ }^{1}$ State Key Laboratory of Oral Diseases and ${ }^{2}$ Department of Head and Neck Oncology Surgery, \\ West China Hospital of Stomatology, Sichuan University, Chengdu, Sichuan; \\ ${ }^{3}$ Department of Forensic Science, School of Basic Medical Sciences, \\ Central South University, Changsha, Hunan; ${ }^{4}$ Department of Head and Neck Oncology, \\ Sichuan Cancer Hospital, Chengdu, Sichuan, P.R. China
}

Received August 21, 2013; Accepted October 22, 2013

DOI: 10.3892/etm.2013.1375

\begin{abstract}
Oxidative stress is significant in numerous types of cancer. Tobacco smoke, an important risk factor for oral squamous cell carcinoma (OSCC), is able to generate reactive oxygen species (ROS) and cause oxidative DNA damage. Superoxide dismutase (SOD) is an endogenous antioxidant enzyme that is critical in limiting the oxidative burden effectively. The purpose of this study was to investigate the effects of the mitochondrial SOD2 and $\mathrm{Cu} / \mathrm{Zn}$ enzyme SOD1 gene polymorphisms on the susceptibility to and clinicopathological characteristics of OSCC, as well as the synergistic effect between these gene polymorphisms and the well-known risk factor of tobacco consumption. Patients with clinically diagnosed OSCC $(n=362)$ and healthy normal individuals $(n=358)$ were investigated for four single nucleotide polymorphisms (SNPs; rs4880, rs5746136, rs1804450 and rs11556620) by polymerase chain reaction-restriction fragment length polymorphism and DNA sequencing methods. Following adjustment for other confounders, no significant difference was observed in the rs5746136 SOD2 SNPs between the patients and controls. However, the incidence of the CT genotype of SOD2 SNP rs4880 was higher in the patients than in normal subjects in the additive model [CT vs. TT; $\mathrm{P}=0.045$; adjusted odds ratio $(\mathrm{AOR})=1.484 ; 95 \%$ confidence interval (CI), 1.009-2.182] and in the dominant model (CT/CC vs. TT; $\mathrm{P}=0.022 ; \mathrm{AOR}=1.559 ; 95 \% \mathrm{CI}, 1.067-2.278)$. For those who smoked, the incidence of the CT genotype of rs4880 increased markedly in the patients compared with the controls in the additive model (CT vs. TT; $\mathrm{P}=0.003 ; \mathrm{AOR}=2.325 ; 95 \% \mathrm{CI}$,
\end{abstract}

Correspondence to: Professor Longjiang Li, Department of Head and Neck Oncology Surgery, West China Hospital of Stomatology, Sichuan University, No. $14,3^{\text {rd }}$ Section, Renmin South Road, Chengdu, Sichuan 610041, P.R. China

E-mail: muzili63@163.com

Key words: superoxide dismutase 1, mitochondrial superoxide dismutase 2 , oral squamous cell carcinoma, gene polymorphisms
$1.330-4.064)$ and in the dominant model (CT/CC vs. TT; $\mathrm{P}=0.001$; $\mathrm{AOR}=2.448$; $95 \% \mathrm{CI}, 1.417-4.230$ ). For SOD1, polymorphisms at rs1804450 and rs11556620 were not present in any of the OSCC or control subjects. The results suggest that SOD2 rs4880 may be involved in the tumorigenesis of OSCC and may be useful as a genetic susceptibility marker for OSCC.

\section{Introduction}

Oral squamous cell carcinoma (OSCC), the sixth most common type of cancer in the world, is a major cause of morbidity and mortality and $>31,000$ patients are newly diagnosed with OSCC in the USA annually (1). It is one of the most prevalent cancers in the human male population worldwide, with approximately two-thirds of all cases occurring in developing countries (2). Despite substantial effort and novel therapeutic developments, the five-year survival rate for OSCC has not appreciably improved over the last two decades $(1,3)$. In China, the risk of morbidity due to OSCC is increasing, and the patients tend to be increasingly younger (4). OSCC is therefore a significant public health threat throughout the world (5).

It is widely accepted that the major risk factor for OSCC is chronic exposure of the oral epithelia to tobacco smoke and alcohol $(6,7)$. Tobacco smoke produces a variety of free radicals, reactive oxygen species (ROS) and reactive nitrogen species (RNS), which cause oxidative damage and oxidative stress through the production of superoxide, hydrogen peroxide and nitric oxide in cells (8). Oxidative stress is significantly involved in several pathological conditions, including cardiovascular disease, neurological disorders, aging and cancer $(8,9)$. Interactions between ROS and RNS are a crucial determinant of the etiology of OSCC, and conversely, antioxidants protect against the cellular and molecular damage caused by these interactions $(10,11)$. Lin et al reported that cigarette smoking, alcohol consumption and betel quid chewing have all been identified as significant causes of oral carcinogenesis, primarily due to their ability to generate ROS and cause oxidative DNA damage (12).

Superoxide dismutases (SODs) are enzymes that catalyze the dismutation of superoxide into oxygen and hydrogen 
peroxide (13). There are three major families of superoxide dismutase, depending on the metal cofactor: $\mathrm{Cu} / \mathrm{Zn}$ (SOD1, OMIM number 147450), which binds copper and zinc; Fe and Mn types (SOD2, OMIM number 147460), which bind either iron or manganese; and the Ni type (SOD3, OMIM number 185490), which binds nickel (14). Mitochondrial SOD2 is an endogenous antioxidant enzyme that is significantly involved in limiting the oxidative burden (15). SOD2 converts superoxide into hydrogen peroxide and oxygen in the mitochondria and plays a major role in protecting cells from oxidative damage (16). A number of studies have demonstrated that the activity and expression of SOD2 changes significantly in patients with oral cancer, and either promotes or suppresses tumor formation (17-19). Therefore, in the present study it was hypothesized that the polymorphic variants of SOD2 may be involved in the development and prognosis of oral cancer. SOD1 is mainly located in the cytoplasm and accounts for $\sim 85 \%$ of the total cellular SOD activity of most mammalian cells (20).

Polymorphic variants of SOD2 have been demonstrated to be factors that may enhance the risk of cancer development (21). The SOD2 SNP rs4880 (T>C) leads to a conformational change in the helical structure of the protein and has been associated with several carcinomas $(9,15,22)$. rs5746136 in the 3'UTR of SOD2 is located 65 bp downstream of the poly A site. It is also $<1 \mathrm{~kb}$ upstream from SP1 and the NF- $\kappa \mathrm{B}$ transcription element sequences (23). To date, studies have rarely been conducted on polymorphisms of $\mathrm{CuZnSOD}$ in oral cancer (24). Therefore, in the present study, two other SNPs [rs1804450, Thr40Ile(C/T) and rs11556620, Asn87Ser(A/G)] were selected for investigation of their association with OSCC. A hospital-based case-control study was conducted to examine whether polymorphisms in rs4880, rs5746136, rs1804450 and rs11556620 SNPs are associated with OSCC. This is a novel study in which variations in SOD are evaluated with regard to OSCC risk.

\section{Material and methods}

Patients and controls. Han Chinese with clinically diagnosed OSCC $(n=362)$ were recruited at the West China Hospital of Stomatology, Sichuan University (Chengdu, China), from December 2009 to March 2011. Han Chinese healthy individuals $(n=358)$ who visited the general health check-up division of the West China Hospital of Stomatology, Sichuan University were recruited as controls.

All of the participants were residents of Sichuan Province, China. All subjects were informed of the detailed study protocol, signed consent forms and were asked to fill out a standardized questionnaire with two trained interviewers. The information required in the questionnaire included: i) Demographic factors, such as age, gender and ethnicity; ii) smoking and alcohol drinking habits (participants were considered as smokers if they had smoked at least one cigarette per day for the past six months or longer and as drinkers if they had drunk $100 \mathrm{ml}$ of alcohol at least twice a week for the past six months); iii) disease, which consisted of systemic diseases or cancer in other sites; and iv) family history of cancer in parents, brothers and sisters. According to the inclusion criteria, participants with systemic diseases and/or a family history of cancer were filtered out by the standardized questionnaire and their medical records, so none of the participants in the study had a disease (except OSCC in the case group), a family history of cancer or the habit of betel nut chewing (there is no habit of betel nut chewing in Sichuan Province). All subjects in the patient group had their disease histopathologically confirmed and were previously untreated patients in the surgery departments. The OSCC information for all patients was collected from their medical records following their surgeries. Ethical approval was granted by the West China Hospital of Stomatology, Sichuan University ethics committee and all patients gave written consent prior to collection of the samples. Characteristics of the case group and control group are presented in Table I and the clinicopathological features of the case group are listed in Table II.

DNA extraction and genotyping. Blood was taken from all participants by peripheral antecubital venous puncture and was stored at $-20^{\circ} \mathrm{C}$ until analysis.

Genomic DNA was extracted using a salting-out method (25). The DNA concentration of each individual sample was measured on a NanoDrop 1000 Spectrophotometer using NanoDrop software, version 2.4.7c (NanoDrop Technologies Inc., Wilmington, DE, USA). The SOD2 and SOD1 genotypes rs4880, rs5746136, rs1804450 and rs11556620 were identified using a polymerase chain reaction-restriction fragment length polymorphism method (PCR-RFLP). The PCR primers were designed according to the reference sequence in the SNP database (dbSNP) of the NCBI (see Table III for primer sequences and reaction conditions). The PCR reaction consisted of $2.5 \mu \mathrm{l}$ 10X PCR buffer, $0.1 \mathrm{mM}$ dNTPs, $1 \mathrm{mM} \mathrm{MgCl}_{2}, 2$ units Taq polymerase (Tiangen, Beijing, China), 10 ng genomic DNA, $0.4 \mathrm{pM}$ each primer and enough $\mathrm{ddH}_{2} \mathrm{O}$ to make the volume up to $25 \mu$ l per reaction.

The four PCR products were digested with 3 units of one of the specific endonucleases, TaqI, BsaWI, AcuI or BtsI, for $4 \mathrm{~h}$ at $60^{\circ} \mathrm{C}$ or $37^{\circ} \mathrm{C}$ (Table III). PCR and digestion products were analyzed by electrophoresis in $7 \%$ or $8 \%$ polyacrylamide gels stained with silver nitrate. To confirm the genotyping results of each genetic polymorphism, $10-15 \%$ of the samples in each genotype group were randomly selected for Sanger sequencing, which was performed by Sangon Biotech (Shanghai, China). The results were $100 \%$ concordant with the RFLP analysis.

Statistical analysis. The differences in the demographic characteristics were compared between the OSCC cases and controls using Student's t-test and $\chi^{2}$ test. Student's t-test was used to compare the difference in age and $\chi^{2}$ test was used to compare the differences in gender and tobacco and alcohol consumption. Models were run under the assumption of additive (AA vs. Aa vs. aa), dominant (AA vs. Aa/aa) or recessive (AA/Aa vs. aa) inheritance. Genotype frequencies of rs 4880 and rs5746136 were compared between the OSCC cases and controls using the unconditional logistic regression. The odds ratios (ORs) with their 95\% confidence intervals (CIs) were estimated by unconditional logistic regression. The adjusted P-values (Pas) were estimated by multiple logistic regression. The adjusted ORs (AORs), with their 95\% CIs, of the association between the genotype frequencies and OSCC susceptibility were estimated by multiple logistic regression 
models after controlling for other covariates such as age, gender, smoking and drinking. The Hardy-Weinberg equilibrium was tested with a goodness of fit $\chi^{2}$ test with one degree of freedom to compare the observed genotype frequencies among the subjects with the expected genotype frequencies calculated byPowerStats v1.2 software (Promega Corporation, Madison, WI, USA). Statistical significance was assumed at $\mathrm{P}<0.05$. SPSS Software, version 11.5 (SPSS, Inc., Chicago, IL, USA) was used for all of the statistical analyses. SHEsis software (http://analysis.bio-x.cn/SHEsisMain.htm) was applied for analysis of linkage disequilibrium (LD) (11).

\section{Results}

The statistical data indicated there were no significant differences in age, gender and drinking between the patient group and the control group ( $\mathrm{P}>0.05)$, but did identify that there were significant differences in smoking $(\mathrm{P}<0.0001$; Table I).

Multiple logistic regression analyses were conducted for rs4880 (additive, CT vs. TT and CC vs. TT; dominant, CT/CC vs. TT; and recessive, CC vs. TT/CT) and rs5746136 (additive, AG vs. GG and AA vs. GG; dominant, AG/AA vs. GG; and recessive, AA vs. GG/GA). For SOD1, polymorphisms at rs1804450 and rs11556620 were not present in any of the OSCC and control subjects, hence these two SNPs were ruled out from subsequent analyses. The AORs and the respective $95 \%$ CIs of the genotype distributions of rs4880 and rs5746136 associated with the susceptibility of OSCC between the case group and control group are presented in Table IV. There was no significant association between genetic polymorphisms of rs5746136 and OSCC in the present study under additive, dominant and recessive models (Table IV). However, there was a significant 1.559 -fold difference in the frequencies of the rs4880 genotypes containing the $\mathrm{C}$ allele between patients and control subjects in the dominant model (CC/CT vs. TT, 95\% CI, 1.067-2.278). Moreover, the CT genotype was associated with a 1.484-fold significantly higher risk (CT vs. TT, 95\% CI, 1.009-2.182) in the additive model. The genotype frequencies of rs4880 were significantly associated with OSCC in males in the additive and dominant models (Table V). In the additive model, the CT genotype was associated with a 1.663-fold significantly higher risk (CT vs. TT, 95\% CI, 1.010-2.739). In the dominant model, the frequencies of the genotypes containing the $\mathrm{C}$ allele had a significant 1.809-fold difference (CC/CT vs. TT, 95\% CI, 1.106-2.960) in males of the OSCC case group compared with the control group (Table V). The data indicate no significant association between genetic polymorphisms of rs5746136 with OSCC among individuals of either gender, nor of rs4880 with OSCC in females.

Furthermore, the $\mathrm{Pa}, \mathrm{AOR}$ and $95 \% \mathrm{CI}$ of genotypic frequencies and OSCC were also estimated among individuals with exposure or non-exposure to tobacco consumption. The genotype frequencies of rs4880 were significantly different between the OSCC patients who smoked and the control participants who smoked in the additive model (CT vs. TT) and dominant model (CC/CT vs. TT; Table VI). In the additive model, the CT genotype was associated with a 2.325-fold greater risk (CT vs. TT, 95\% CI, 1.330-4.064). In the dominant model, the frequencies of the genotypes containing the $\mathrm{C}$ allele were significantly higher (by 2.448 -fold) in patients with OSCC
Table I. Characteristics of the case and control groups.

\begin{tabular}{lccc}
\hline Characteristics & Case $(\mathrm{n}=362)$ & Control (n=358) & P-value $^{\mathrm{a}}$ \\
\hline $\begin{array}{l}\text { Age (years) } \\
\text { Range }\end{array}$ & $19-87$ & $28-81$ & \\
Mean + SD & $60.89 \pm 11.57$ & $59.98 \pm 11.41$ & 0.786 \\
Gender, n (\%) & & & \\
Males & $227(62.7)$ & $205(57.3)$ & \\
Females & $135(37.3)$ & $153(42.7)$ & 0.136 \\
Smoking, n (\%) & & & \\
Yes & $254(70.2)$ & $158(44.1)$ & \\
No & $108(29.8)$ & $200(55.9)$ & $<0.0001^{\mathrm{b}}$ \\
Drinking, n (\%) & & & \\
Yes & $208(57.5)$ & $184(51.4)$ & \\
No & $154(42.5)$ & $174(48.6)$ & 0.102 \\
\hline
\end{tabular}

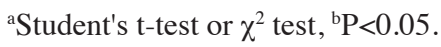

Table II. Clinicopathological features of the case group $(n=362)$.

\begin{tabular}{|c|c|c|}
\hline Characteristics & $\mathrm{n}$ & $\%$ \\
\hline \multicolumn{3}{|l|}{ Site } \\
\hline Tongue & 124 & 34.3 \\
\hline Buccal mucosa & 99 & 27.3 \\
\hline Gingiva & 63 & 17.4 \\
\hline Floor of mouth & 41 & 11.3 \\
\hline Palate & 22 & 6.1 \\
\hline Lip & 11 & 3.0 \\
\hline Maxillary sinus & 2 & 0.6 \\
\hline \multicolumn{3}{|l|}{ Tumor Size } \\
\hline $\mathrm{T} 1+\mathrm{T} 2$ & 208 & 57.5 \\
\hline $\mathrm{T} 3+\mathrm{T} 4$ & 154 & 42.5 \\
\hline \multicolumn{3}{|l|}{ Lymph node metastases } \\
\hline No & 233 & 64.4 \\
\hline $\mathrm{N}+$ & 129 & 35.6 \\
\hline \multicolumn{3}{|l|}{ Clinical stage } \\
\hline I & 58 & 16.0 \\
\hline II & 101 & 27.9 \\
\hline III & 71 & 19.6 \\
\hline IV & 132 & 36.5 \\
\hline \multicolumn{3}{|l|}{ Pathological stage } \\
\hline SCC I (highly differentiated) & 253 & 69.9 \\
\hline SCC II (moderately differentiated) & 99 & 27.3 \\
\hline SCC III (poorly differentiated) & 10 & 2.8 \\
\hline
\end{tabular}

SCC, squamous cell carcinoma.

who smoked compared with those in healthy controls who smoked (CC/CT vs. TT, 95\% CI, 1.417-4.230). However, no association was identified between the genetic polymorphism 
Table III. PCR primers and restriction enzymes used for amplification of the corresponding fragments.

\begin{tabular}{|c|c|c|c|c|c|c|c|}
\hline $\begin{array}{l}\text { PCR } \\
\text { products }\end{array}$ & $\mathrm{SNP}(\mathrm{rs} \#)$ & Polymorphism & Primer sequence & $\begin{array}{l}\text { Annealing } \\
\text { temp }\left({ }^{\circ} \mathrm{C}\right)\end{array}$ & $\begin{array}{l}\text { Amplicon } \\
\text { size (bp) }\end{array}$ & $\begin{array}{l}\text { Restriction } \\
\text { enzyme }\end{array}$ & $\begin{array}{c}\text { Restriction } \\
\text { products (bp) }\end{array}$ \\
\hline SOD2 & rs4880 & Val16Ala(T/C) & $\begin{array}{l}\text { GCTGTGCTTTCTCGTCTTCAG } \\
\text { TGGTACTTCTCCTCGGTGACG }\end{array}$ & 58 & 208 & BsaW1 & $\begin{array}{l}41 / 167 \\
\text { or } 208\end{array}$ \\
\hline SOD2 & rs5746136 & $(\mathrm{C} / \mathrm{T})$ & $\begin{array}{l}\text { AAAAACCACTGGGTGACATCTAC } \\
\text { AAGGGAAACACTCGGCTTTCT }\end{array}$ & 60 & 94 & TaqI & $\begin{array}{l}42 / 52 \\
\text { or } 94\end{array}$ \\
\hline SOD1 & rs1804450 & Thr40Ile(C/T) & $\begin{array}{l}\text { CACAACACCCACCTGCTGTATTA } \\
\text { GTGAAGGTGTGGGGAAGCATT }\end{array}$ & 57 & 94 & AcuI & $\begin{array}{l}41 / 53 \\
\text { or } 94\end{array}$ \\
\hline SOD1 & rs11556620 & Asn87Ser(A/G) & $\begin{array}{l}\text { TTAGTGGCATCAGCCCTAATCCAT } \\
\text { ATAGACACATCGGCCACACCA }\end{array}$ & 60 & 105 & BtsI & $\begin{array}{l}36 / 69 \\
\text { or } 105\end{array}$ \\
\hline
\end{tabular}

PCR, polymerase chain reaction; SOD, superoxide dismutase.

Table IV. Comparison of the genotype frequencies of the SOD 2 polymorphisms between OSCC patients and controls.

\begin{tabular}{|c|c|c|c|c|c|c|c|c|}
\hline SNP & Model/Allele & Genotype & $\begin{array}{c}\text { Case }(\%) \\
n=362\end{array}$ & $\begin{array}{c}\text { Control }(\%) \\
n=358\end{array}$ & P-value ${ }^{a}$ & $\begin{array}{c}\mathrm{OR}^{\mathrm{b}} \\
(95 \% \mathrm{CI})\end{array}$ & $\mathrm{Pa}^{\mathrm{c}}$ & $\begin{array}{c}\mathrm{AOR}^{\mathrm{d}} \\
(95 \% \mathrm{CI})\end{array}$ \\
\hline \multirow[t]{7}{*}{ rs 4880} & \multirow[t]{3}{*}{ Additive } & TT & $272(75.1)$ & $296(82.7)$ & & 1.00 (Ref) & & \\
\hline & & CT & $83(23.0)$ & $61(17.0)$ & 0.037 & $\begin{array}{c}1.481 \\
(1.024-2.142)\end{array}$ & $0.045^{\mathrm{e}}$ & $\begin{array}{c}1.484 \\
(1.009-2.182)\end{array}$ \\
\hline & & $\mathrm{CC}$ & 7 (1.9) & $1(0.3)$ & 0.033 & $\begin{array}{c}7.618 \\
(0.931-62.316)\end{array}$ & 0.121 & $\begin{array}{c}5.388 \\
(0.642-45.216)\end{array}$ \\
\hline & \multirow[t]{2}{*}{ Dominant } & $\mathrm{TT}$ & $272(75.1)$ & $296(82.7)$ & & 1.00 (Ref) & & \\
\hline & & $\mathrm{CT} / \mathrm{CC}$ & $90(24.9)$ & $62(17.3)$ & 0.013 & $\begin{array}{c}1.580 \\
(1.099-2.271)\end{array}$ & $0.022^{\mathrm{e}}$ & $\begin{array}{c}1.559 \\
(1.067-2.278)\end{array}$ \\
\hline & \multirow[t]{2}{*}{ Recessive } & $\mathrm{TT} / \mathrm{CT}$ & $355(98.1)$ & 357 (99.7) & & & & \\
\hline & & $\mathrm{CC}$ & 7 (1.9) & $1(0.3)$ & 0.069 & $\begin{array}{c}7.039 \\
(0.862-57.510)\end{array}$ & 0.138 & $\begin{array}{c}4.988 \\
(0.595-41.803)\end{array}$ \\
\hline \multirow[t]{7}{*}{ rs5746136 } & \multirow[t]{3}{*}{ Additive } & GG & $99(27.3)$ & 103 (28.8) & & 1.00 (Ref) & & \\
\hline & & $\mathrm{AG}$ & $172(47.5)$ & $180(50.3)$ & 0.974 & $\begin{array}{c}0.994 \\
(0.703-1.405)\end{array}$ & 0.960 & $\begin{array}{c}1.009 \\
(0.703-1.449)\end{array}$ \\
\hline & & $\mathrm{AA}$ & $91(25.1)$ & 75 (20.9) & 0.267 & $\begin{array}{c}1.262 \\
(0.836-1.905)\end{array}$ & 0.418 & $\begin{array}{c}1.194 \\
(0.778-1.833)\end{array}$ \\
\hline & \multirow[t]{2}{*}{ Dominant } & GG & 99 (27.3) & 103 (28.8) & & 1.00 (Ref) & & \\
\hline & & AG/AA & $263(72.7)$ & 255 (71.2) & 0.671 & $\begin{array}{c}1.073 \\
(0.775-1.148)\end{array}$ & 0.715 & $\begin{array}{c}1.065 \\
(0.759-1.496)\end{array}$ \\
\hline & \multirow[t]{2}{*}{ Recessive } & $\mathrm{GG} / \mathrm{AG}$ & $271(74.9)$ & 283 (79.1) & & 1.00 (Ref) & & \\
\hline & & $\mathrm{AA}$ & $91(25.1)$ & 75 (20.9) & 0.182 & $\begin{array}{c}1.267 \\
(0.895-1.795)\end{array}$ & 0.354 & $\begin{array}{c}1.187 \\
(0.826-1.839)\end{array}$ \\
\hline
\end{tabular}

aP-values were estimated by unconditional logistic regression. ${ }^{6}$ The odds ratios (ORs) with their $95 \%$ confidence intervals (CIs) were estimated by unconditional logistic regression. ${ }^{\mathrm{C} A d j u s t e d ~ P-v a l u e s ~(P a s) ~ w e r e ~ e s t i m a t e d ~ b y ~ m u l t i p l e ~ l o g i s t i c ~ r e g r e s s i o n ~ a f t e r ~ c o n t r o l l i n g ~ f o r ~ a g e, ~ g e n d e r, ~}$ smoking and drinking. ${ }^{\mathrm{T}}$ The adjusted ORs (AORs) with their $95 \%$ CIs were estimated by multiple logistic regression models after controlling for age, gender, smoking and drinking. ${ }^{e} \mathrm{P}<0.05$. SOD, superoxide dismutase; OSSC, oral squamous cell carcinoma, SNP, single nucleotide polymorphism; Ref, reference.

of rs4880 and OSCC in non-smokers after adjustment for other covariates (Table VI). Moreover, according to multiple logistic regression models after controlling for relevant factors, no significant association was identified between the genetic polymorphisms of rs5746136 and OSCC, regardless of whether individuals consumed tobacco. 
Table V. Comparison of the genotype frequencies of the SOD 2 polymorphisms among males and females.

A. Among males (case, $\mathrm{n}=227$ and control, $\mathrm{n}=205$ )

\begin{tabular}{|c|c|c|c|c|c|c|c|c|}
\hline SNP & Model/Allele & Genotype & Case (\%) & Control (\%) & P-value ${ }^{a}$ & OR $(95 \% \mathrm{CI})^{\mathrm{b}}$ & $\mathrm{Pa}^{\mathrm{c}}$ & AOR $(95 \% \text { CI })^{\mathrm{d}}$ \\
\hline \multirow[t]{7}{*}{ rs4880 } & \multirow[t]{3}{*}{ Additive } & TT & $166(73.1)$ & $170(82.9)$ & & 1.00 (Ref) & & \\
\hline & & $\mathrm{CT}$ & $56(24.7)$ & $35(17.1)$ & 0.040 & $\begin{array}{c}1.639 \\
(1.021-2.631)\end{array}$ & $0.045^{\mathrm{e}}$ & $\begin{array}{c}1.663 \\
(1.010-2.739)\end{array}$ \\
\hline & & $\mathrm{CC}$ & $5(2.2)$ & $0(0)$ & - & - & - & - \\
\hline & \multirow[t]{2}{*}{ Dominant } & TT & $166(73.1)$ & $170(82.9)$ & & 1.00 (Ref) & & \\
\hline & & $\mathrm{CT} / \mathrm{CC}$ & $61(26.9)$ & $35(17.1)$ & 0.014 & $\begin{array}{c}1.785 \\
(1.118-2.848)\end{array}$ & $0.018^{\mathrm{e}}$ & $\begin{array}{c}1.809 \\
(1.106-2.960)\end{array}$ \\
\hline & \multirow[t]{2}{*}{ Recessive } & $\mathrm{TT} / \mathrm{CT}$ & $222(97.8)$ & $205(100)$ & & 1.00 (Ref) & & \\
\hline & & $\mathrm{CC}$ & $5(2.2)$ & $0(0)$ & - & - & - & - \\
\hline \multirow[t]{7}{*}{ rs5746136 } & \multirow[t]{3}{*}{ Additive } & GG & $64(28.2)$ & $58(28.3)$ & & 1.00 (Ref) & & \\
\hline & & $\mathrm{AG}$ & $103(45.4)$ & $99(48.3)$ & 0.798 & $\begin{array}{c}0.943 \\
(0.601-1.478)\end{array}$ & 0.944 & $\begin{array}{c}1.020 \\
(0.594-1.751)\end{array}$ \\
\hline & & AA & $60(26.4)$ & $48(23.4)$ & 0.638 & $\begin{array}{c}1.133 \\
(0.674-1.905)\end{array}$ & 0.601 & $\begin{array}{c}0.882 \\
(0.550-1.413)\end{array}$ \\
\hline & \multirow[t]{2}{*}{ Dominant } & GG & $64(28.2)$ & $58(28.3)$ & & 1.00 (Ref) & & \\
\hline & & AG/AA & $163(71.8)$ & $147(71.7)$ & 0.982 & $\begin{array}{c}1.005 \\
(0.661-1.529)\end{array}$ & 0.739 & $\begin{array}{c}0.928 \\
(0.598-1.440)\end{array}$ \\
\hline & \multirow[t]{2}{*}{ Recessive } & $\mathrm{GG} / \mathrm{AG}$ & 167 (73.6) & $157(76.8)$ & & 1.00 (Ref) & & \\
\hline & & AA & $60(26.4)$ & $48(23.4)$ & 0.470 & $\begin{array}{c}1.175 \\
(0.759-1.820)\end{array}$ & 0.672 & $\begin{array}{c}1.103 \\
(0.701-1.735)\end{array}$ \\
\hline
\end{tabular}

B. Among females (case, $\mathrm{n}=135$ and control, $\mathrm{n}=153$ )

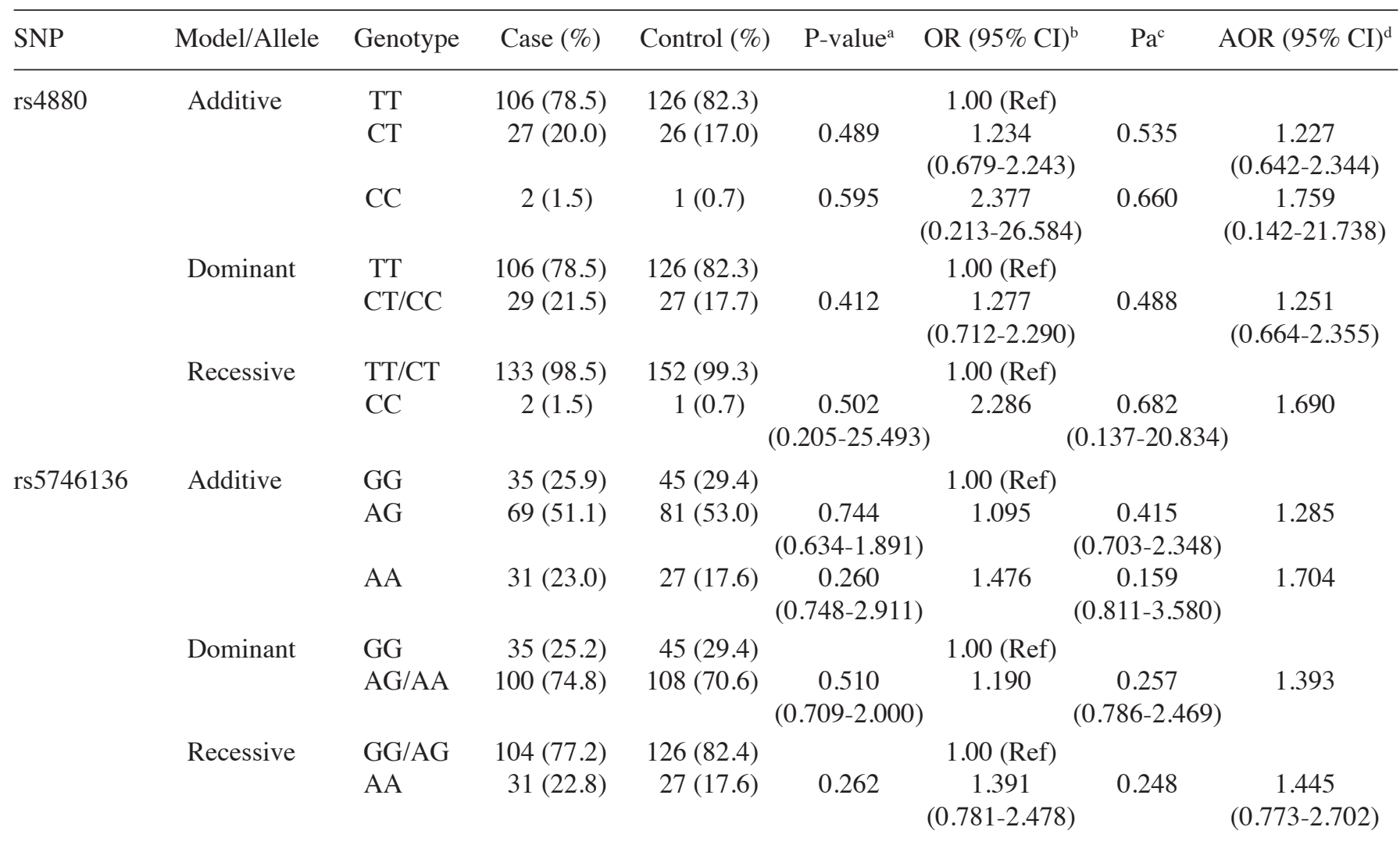

aP-values were estimated by unconditional logistic regression. ${ }^{6}$ The odds ratios (ORs) with their 95\% confidence intervals (CIs) were estimated by unconditional logistic regression. ${ }^{\mathrm{c} A d j u s t e d ~ P-v a l u e s ~(P a s) ~ w e r e ~ e s t i m a t e d ~ b y ~ m u l t i p l e ~ l o g i s t i c ~ r e g r e s s i o n ~ a f t e r ~ c o n t r o l l i n g ~ f o r ~ a g e, ~ g e n d e r, ~}$ smoking and drinking. ${ }^{\mathrm{d} T h e}$ adjusted ORs (AORs) with their $95 \%$ CIs were estimated by multiple logistic regression models after controlling for age, smoking and drinking. ${ }^{\mathrm{e}} \mathrm{P}<0.05$. SOD, superoxide dismutase; OSSC, oral squamous cell carcinoma; SNP, single nucleotide polymorphism; Ref, reference. 
Table VI. Comparison of the genotype frequencies of the SOD 2 polymorphisms among smokers and non-smokers.

A. Among smokers (case, $\mathrm{n}=254$ and control, $\mathrm{n}=158$ )

\begin{tabular}{|c|c|c|c|c|c|c|c|c|}
\hline SNP & Model/Allele & Genotype & Case $(\%)$ & Control (\%) & P-value ${ }^{a}$ & OR $(95 \% \text { CI })^{b}$ & $\mathrm{~Pa}^{\mathrm{c}}$ & $\operatorname{AOR}(95 \% \mathrm{CI})^{\mathrm{d}}$ \\
\hline \multirow[t]{7}{*}{ rs4880 } & \multirow[t]{3}{*}{ Additive } & $\mathrm{TT}$ & $185(72.8)$ & $135(85.4)$ & & 1.00 (Ref) & & \\
\hline & & $\mathrm{CT}$ & $63(24.8)$ & $22(13.9)$ & 0.006 & $\begin{array}{c}2.090 \\
(1.225-3.563)\end{array}$ & $0.003^{\mathrm{e}}$ & $\begin{array}{c}2.325 \\
(1.330-4.064)\end{array}$ \\
\hline & & $\mathrm{CC}$ & $6(2.4)$ & $1(0.7)$ & 0.138 & $\begin{array}{c}4.378 \\
(0.521-36.792)\end{array}$ & 0.134 & $\begin{array}{c}5.404 \\
(0.594-49.189)\end{array}$ \\
\hline & \multirow[t]{2}{*}{ Dominant } & $\mathrm{TT}$ & $185(72.8)$ & $135(85.4)$ & & 1.00 (Ref) & & \\
\hline & & $\mathrm{CT} / \mathrm{CC}$ & $69(27.2)$ & $23(14.6)$ & 0.003 & $\begin{array}{c}2.189 \\
(1.300-3.688)\end{array}$ & $0.001^{\mathrm{e}}$ & $\begin{array}{c}2.448 \\
(1.417-4.230)\end{array}$ \\
\hline & \multirow[t]{2}{*}{ Recessive } & $\mathrm{TT} / \mathrm{CT}$ & $248(97.6)$ & 157 (99.3) & & $1.00(\operatorname{Ref})$ & & \\
\hline & & $\mathrm{CC}$ & $6(2.4)$ & $1(0.7)$ & 0.187 & $\begin{array}{c}3.789 \\
(0.453-31.849)\end{array}$ & 0.181 & $\begin{array}{c}4.485 \\
(0.498-40.420)\end{array}$ \\
\hline \multirow[t]{7}{*}{ rs5746136 } & \multirow[t]{3}{*}{ Additive } & GG & $68(26.8)$ & $47(29.7)$ & & 1.00 (Ref) & & \\
\hline & & $\mathrm{AG}$ & $121(47.6)$ & $70(44.3)$ & 0.462 & $\begin{array}{c}1.195 \\
(0.744-1.920)\end{array}$ & 0.335 & $\begin{array}{c}1.272 \\
(0.780-2.074)\end{array}$ \\
\hline & & AA & $65(25.6)$ & $41(25.9)$ & 0.740 & $\begin{array}{c}1.096 \\
(0.639-1.879)\end{array}$ & 0.606 & $\begin{array}{c}1.157 \\
(0.665-2.013)\end{array}$ \\
\hline & \multirow[t]{2}{*}{ Dominant } & GG & $68(26.8)$ & $47(29.7)$ & & 1.00 (Ref) & & \\
\hline & & AG/AA & $186(73.2)$ & $111(70.3)$ & 0.513 & $\begin{array}{c}1.158 \\
(0.746-1.798)\end{array}$ & 0.372 & $\begin{array}{c}1.229 \\
(0.782-1.932)\end{array}$ \\
\hline & \multirow[t]{2}{*}{ Recessive } & GG/AG & $189(74.4)$ & $117(74.1)$ & & 1.00 (Ref) & & \\
\hline & & AA & $65(25.6)$ & $41(25.9)$ & 0.935 & $\begin{array}{c}0.981 \\
(0.623-1.545)\end{array}$ & 0.993 & $\begin{array}{c}0.998 \\
(0.626-1.592)\end{array}$ \\
\hline
\end{tabular}

B. Among non-smokers (case, $\mathrm{n}=108$ and control, $\mathrm{n}=200$ )

\begin{tabular}{|c|c|c|c|c|c|c|c|c|}
\hline SNP & Model/Allele & Genotype & Case $(\%)$ & Control (\%) & P-value ${ }^{a}$ & OR $(95 \% \mathrm{CI})^{\mathrm{b}}$ & $\mathrm{Pa}^{\mathrm{c}}$ & $\operatorname{AOR}(95 \% \mathrm{CI})^{\mathrm{d}}$ \\
\hline \multirow[t]{7}{*}{ rs4880 } & Additive & TT & 87 (80.6) & $161(80.5)$ & & 1.00 (Ref) & & \\
\hline & & $\mathrm{CT}$ & $20(18.5)$ & $39(19.5)$ & 0.864 & $\begin{array}{c}0.949 \\
(0.521-1.727)\end{array}$ & 0.962 & $\begin{array}{c}1.016 \\
(0.540-1.911)\end{array}$ \\
\hline & & $\mathrm{CC}$ & $1(0.9)$ & $0(0)$ & - & - & - & - \\
\hline & Dominant & TT & 87 (80.6) & $161(80.5)$ & & 1.00 (Ref) & & \\
\hline & & $\mathrm{CT} / \mathrm{CC}$ & $21(19.4)$ & $39(19.5)$ & 0.991 & $\begin{array}{c}0.996 \\
(0.552-1.800)\end{array}$ & 0.848 & $\begin{array}{c}1.064 \\
(0.569-1.987)\end{array}$ \\
\hline & Recessive & $\mathrm{TT} / \mathrm{CT}$ & 107 (99.1) & $200(100)$ & & 1.00 (Ref) & & \\
\hline & & $\mathrm{CC}$ & $1(0.9)$ & $0(0)$ & - & - & - & - \\
\hline \multirow[t]{7}{*}{ rs5746136 } & Additive & GG & $31(28.7)$ & $56(28.0)$ & & 1.00 (Ref) & & \\
\hline & & $\mathrm{AG}$ & $51(47.2)$ & $110(55.0)$ & 0.527 & $\begin{array}{c}0.838 \\
(0.483-1.452)\end{array}$ & 0.462 & $\begin{array}{c}0.804 \\
(0.448-1.440)\end{array}$ \\
\hline & & AA & $26(24.1)$ & $34(17.0)$ & 0.346 & $\begin{array}{c}1.381 \\
(0.705-2.708)\end{array}$ & 0.303 & $\begin{array}{c}1.457 \\
(0.712-2.978)\end{array}$ \\
\hline & Dominant & GG & $31(28.7)$ & $56(28.0)$ & & 1.00 (Ref) & & \\
\hline & & AG/AA & 77 (71.3) & $144(72.0)$ & 0.896 & $\begin{array}{c}0.966 \\
(0.575-1.623)\end{array}$ & 0.862 & $\begin{array}{c}0.952 \\
(0.550-1.649)\end{array}$ \\
\hline & Recessive & $\mathrm{GG} / \mathrm{AG}$ & $82(75.9)$ & $166(83.0)$ & & 1.00 (Ref) & & \\
\hline & & AA & $26(24.1)$ & $34(17.0)$ & 0.135 & $\begin{array}{c}1.548 \\
(0.871-2.751)\end{array}$ & 0.098 & $\begin{array}{c}1.676 \\
(0.909-3.091)\end{array}$ \\
\hline
\end{tabular}

a P-values were estimated by unconditional logistic regression. ${ }^{\text {b}}$ The odds ratios (ORs) with their 95\% confidence intervals (CIs) were estimated by unconditional logistic regression. ${ }^{\mathrm{c} A d j u s t e d ~ P-v a l u e s ~(P a s) ~ w e r e ~ e s t i m a t e d ~ b y ~ m u l t i p l e ~ l o g i s t i c ~ r e g r e s s i o n ~ a f t e r ~ c o n t r o l l i n g ~ f o r ~ a g e, ~ g e n d e r, ~}$ smoking and drinking. ${ }^{\mathrm{d}}$ The adjusted ORs (AORs) with their $95 \%$ CIs were estimated by multiple logistic regression models after controlling for age, gender and drinking. ${ }^{\mathrm{e}} \mathrm{P}<0.05$. SOD, superoxide dismutase; OSSC, oral squamous cell carcinoma; SNP, single nucleotide polymorphism; Ref, reference. 
The LD values for rs4880 and rs5746136 were evaluated using SHEsis. No significant LD was observed for the two loci; the $r^{2}$ value was 0.009 and the $D^{\prime}$ value was 0.275 . PowerStats software analysis revealed no large deviations $(\mathrm{P}>0.05)$ from the Hardy-Weinberg equilibrium for these two loci (rs4880, $\mathrm{P}=0.87515$; rs574616, $\mathrm{P}=0.58383$ ).

The statistical power in this study was $>80 \%$, with an estimated odds ratio of 2.0 (rs4880, 86\%; rs5746136, 82.2\%).

\section{Discussion}

OSCC is the most common type of malignancy of the oral cavity (26). In the past 30 years, the 5-year survival rate for patients with OSCC has not been improved substantially ( $50 \%)$ (27). To date, the most effective treatment for OSCC is extensive surgical resection, with radiotherapy and/or chemotherapy prior to or following surgery $(28,29)$. However, patients are frequently diagnosed at late stages. Gene therapy is also attracting more attention from an increasing number of researchers. The present study attempted to discover suitable novel biomarkers or genes which reflect the physiological state and changes of cells prior to or during the disease process to offer early and accurate prediction and diagnosis for patients with OSCC, particularly in early-stage OSCC (30).

The global prevalence of chronic diseases such as cancer, diabetes mellitus, hypertension, atherosclerosis and Alzheimer's disease is increasing year on year. A number of these diseases are associated with oxidative stress (19) and for numerous cancers, oxidative stress is significantly involved. ROS influence apoptosis, tumor proliferation or promotion (16,31). Of the antioxidative enzymes, SOD2 is essential in the defense against mitochondrial superoxide radicals (32). Successive action of SOD2 detoxifies ROS (33). SOD2 dismutates the superoxide anion into one oxygen molecule and one hydrogen peroxide molecule, which is detoxified into water by glutathione peroxidase (34). In a previous study, intracellular ROS produced by the knockdown of SOD2 enhanced HIF-1 $\alpha$, which is a central regulator that controls the hypoxic response of mammalian cells through the induction of various target genes. Its expression contributes to the development and malignant progression of OSCC (35). In another study, the immunohistochemical staining intensities of SOD2 protein were demonstrated to be higher in well-differentiated OSCCs than in their normal cells of origin (normal oral squamous cells from the basal and spinous layer of oral mucosal tissue) by the examination of oral squamous cell carcinoma biopsies (19). Thus, the published studies suggest that elevated tumor SOD2 levels are associated with enhanced invasiveness of oral cancer (19). Few studies on SOD2 SNPs have been reported in association with oral cancer. Hakenewerth et al suggested that the SOD2 rs4342445 A allele was associated with $30 \%$ greater odds for oral cavity tumors in European- and African-American subjects (24). The SOD2 SNP rs4880 (T>C) in the gene sequence leads to a conformational change in the helical structure of the protein (36). The enzyme is less efficient for transport into mitochondria than the Ala allele (23). This allows Ala-SOD2 to be more efficiently imported into the mitochondrial matrix and to generate more active SOD2 compared with the Val variant (22). Carriage of one or two Ala-SOD2 alleles is reportedly associated with a greater risk of cancer. In a previous study, the Ala variant of SOD2 increased the risk of gastric cancer by altering the activity or content of SOD2 to lower its ability to detoxify ROS (22). A study has suggested that higher antioxidant activity due to an Ala variant of rs4880 in SOD2 may cause poorer survival following cyclophosphamide-containing breast cancer chemotherapy (37). In another study, patients with breast cancer and the high-activity genotype Ala/Ala in the SOD2 rs4880 SNP who were treated with regimens containing cyclophosphamide, but not with hormonal regimens, had significantly poorer prospects for survival than patients with low activity Val alleles (15). Yi et al reported that the SOD1-7958A/- genotype and -16Ala/- genotype, alcohol consumption, a family history of gastric cancer and Helicobacter pylori infection may all be risk factors for gastric cancer in the Han Chinese population (22). Xu et al hypothesized that people who carry the SOD2 rs4880 Ala allele may have active catalysis, resulting in the accumulation of hydrogen peroxide and secondary ROS generation (38).

In the present study, the common SNP for SOD2, rs5746136 C/G, was selected, as well as two other SNPs (rs1804450 and rs11556620 in SOD1) for evaluation of their association with susceptibility to OSCC in comparison with controls. The selection criteria for these three SNPs were that they are located in the coding region of SOD1, and their single-nucleotide changes would lead to an amino acid substitution. To the best of our knowledge, this is the first study to explore the polymorphisms of rs1804450 Thr/Ile and rs11556620 Asn/Ser that do not have genotype data available from the dbSNP of the NCBI. However, neither of these polymorphisms was identified in the members of the Sichuan Province Han population included in the present study.

In this study, no significant difference was observed in the genotype and allele frequencies of rs5746136 SNPs between the patients and controls. However, the $\mathrm{C}$ content of rs4880 was significantly higher in OSCC patients, particularly in the OSCC patients who smoked and in male OSCC patients compared with the control group. The $\mathrm{C}$ allele frequency of rs4880 was significantly higher in OSCC patients compared with normal subjects (CC/CT vs. TT, $\mathrm{AOR}=1.559,95 \% \mathrm{CI}$, 1.067-2.278). The $\mathrm{C}$ allele frequency was also associated with a significantly higher risk of OSCC compared with the TT genotype in smokers (CC/CT vs. TT, AOR $=2.448,95 \% \mathrm{CI}$, 1.417-4.230). Cigarette smoke is one of the most important free radical-generating carcinogens. It was hypothesized that smoking and more highly active SOD2 ( $\mathrm{C}$ allele) may lead to the accumulation of higher levels of hydrogen peroxide and secondary ROS generation, which increases the risk of DNA damage and OSCC. Nevertheless, whether intracellular ROS levels are changed in parallel with the SOD2 rs4880 genotypes in the OSCC microenvironment requires further investigation. The results of the present study corresponded well with other studies on tumors, such as adult brain tumors $(\mathrm{OR}=2.0$; 95\% CI, 1.0-4.2) (9), prostate cancer $(\mathrm{OR}=1.44 ; 95 \% \mathrm{CI}$, 1.1-1.9) (39), gastric cancer (OR=2.04; 95\% CI, 1.19-3.49) (22) and breast cancer (40).

In conclusion, the present study provides the first evidence that a SOD2 polymorphism (C allele of rs4880) may convey a genetic susceptibility to OSCC in a Han Chinese population, but the rs5746136 polymorphism of SOD2 and the SOD1 poly- 
morphisms did not. These results were consistent with previous studies of other types of cancer. This suggests that rs4880 of SOD2 may be a potential marker for disease diagnosis, further supporting the hypothesis that SOD2 is important in carcinogenesis. Further studies with larger samples in diverse ethnic populations are required to verify these conclusions.

\section{Acknowledgements}

This study was supported by National Natural Science Foundation of China (grant no. 30973345 and 81172578).

\section{References}

1. Jemal A, Siegel R, Ward E, et al: Cancer statistics, 2008. CA Cancer J Clin 58: 71-96, 2008

2. Kessler P, Grabenbauer G, Leher A, Bloch-Birkholz A, Vairaktaris E and Neukam FW: Neoadjuvant and adjuvant therapy in patients with oral squamous cell carcinoma: Long-term survival in a prospective, non-randomized study. Br J Oral Maxillofac Surg 46: 1-5, 2008.

3. Jemal A, Bray F, Center MM, Ferlay J, Ward E and Forman D: Global cancer statistics. CA Cancer J Clin 61: 69-90, 2011.

4. Yang L, Parkin DM, Ferlay J, Li L and Chen Y: Estimates of cancer incidence in China for 2000 and projections for 2005. Cancer Epidemiol Biomarkers Prev 14: 243-250, 2005.

5. Sankaranarayanan R, Masuyer E, Swaminathan R, Ferlay J and Whelan S: Head and neck cancer: a global perspective on epidemiology and prognosis. Anticancer Res 18: 4779-4786, 1998.

6. Lazarus P and Park JY: Metabolizing enzyme genotype and risk for upper aerodigestive tract cancer. Oral Oncol 36: 421-431, 2000.

7. Schwartz SM, Doody DR, Fitzgibbons ED, Ricks S, Porter PL and Chen C: Oral squamous cell cancer risk in relation to alcoho consumption and alcohol dehydrogenase-3 genotypes. Cancer Epidemiol Biomarkers Prev 10: 1137-1144, 2001.

8. Ames BN and Gold LS: Endogenous mutagens and the causes of aging and cancer. Mutat Res 250: 3-16, 1991.

9. Rajaraman P, Hutchinson A, Rothman N, et al: Oxidative response gene polymorphisms and risk of adult brain tumors. Neuro Oncol 10: 709-715, 2008.

10. Korde SD, Basak A, Chaudhary M, Goyal M and Vagga A Enhanced nitrosative and oxidative stress with decreased total antioxidant capacity in patients with oral precancer and oral squamous cell carcinoma. Oncology 80: 382-389, 2011.

11. Li Z, Zhang Z, He Z, Tang W, Li T, Zeng Z, He L and Shi Y: A partition-ligation-combination-subdivision EM algorithm for haplotype inference with multiallelic markers: update of the SHEsis (http://analysis.bio-x.cn). Cell Res 19: 519-523, 2009.

12. Lin WJ, Jiang RS, Wu SH, Chen FJ and Liu SA: Smoking, alcohol, and betel quid and oral cancer: a prospective cohort study. J Oncol 2011: 525976, 2011.

13. Borgstahl GE, Parge HE, Hickey MJ, et al: Human mitochondrial manganese superoxide dismutase polymorphic variant Ile58Thr reduces activity by destabilizing the tetrameric interface. Biochemistry 35: 4287-4297, 1996.

14. McCord JM and Fridovich I: Superoxide dismutase: the first twenty years (1968-1988). Free Radic Biol Med 5: 363-369, 1988.

15. Hubackova M, Vaclavikova R, Ehrlichova M, et al: Association of superoxide dismutases and $\mathrm{NAD}(\mathrm{P}) \mathrm{H}$ quinone oxidoreductases with prognosis of patients with breast carcinomas. Int J Cancer 130: 338-348, 2012.

16. Valko M, Leibfritz D, Moncol J, Cronin MT, Mazur M and Telser J: Free radicals and antioxidants in normal physiological functions and human disease. Int J Biochem Cell Biol 39: 44-84, 2007.

17. Kurokawa H, Sakimoto M, Yamashita Y, Murata T and Kajiyama M: Manganese superoxide dismutase (Mn-SOD) correlates with prognosis of patients with oral squamous cell carcinoma. Fukuoka Igaku Zasshi 89: 321-327, 1998.

18. Terakado N, Shintani S, Nakahara Y, et al: Expression of $\mathrm{Cu}, \mathrm{Zn}-\mathrm{SOD}, \mathrm{Mn}-\mathrm{SOD}$ and GST-pi in oral cancer treated with preoperative radiation therapy. Oncol Rep 7: 1113-1117, 2000.
19. Yang J, Lam EW, Hammad HM, Oberley TD and Oberley LW: Antioxidant enzyme levels in oral squamous cell carcinoma and normal human oral epithelium. J Oral Pathol Med 31: 71-77, 2002.

20. Marklund SL: Extracellular superoxide dismutase and other superoxide dismutase isoenzymes in tissues from nine mammalian species. Biochem J 222: 649-655, 1984.

21. Kinnula VL and Crapo JD: Superoxide dismutases in malignant cells and human tumors. Free Radic Biol Med 36: 718-744, 2004.

22. Yi JF, Li YM, Liu T, et al: Mn-SOD and CuZn-SOD polymorphisms and interactions with risk factors in gastric cancer. World J Gastroenterol 16: 4738-4746, 2010.

23. Wiener HW, Perry RT, Chen Z, Harrell LE and Go RC: A polymorphism in SOD2 is associated with development of Alzheimer's disease. Genes Brain Behav 6: 770-775, 2007.

24. Hakenewerth AM, Millikan RC, Rusyn I, et al: Joint effects of alcohol consumption and polymorphisms in alcohol and oxidative stress metabolism genes on risk of head and neck cancer. Cancer Epidemiol Biomarkers Prev 20: 2438-2449, 2011.

25. Miller SA, Dykes DD and Polesky HF: A simple salting out procedure for extracting DNA from human nucleated cells. Nucleic Acids Res 16: 1215, 1988.

26. Jemal A: Global burden of cancer: opportunities for prevention. Lancet 380: 1797-1799, 2012.

27. Yu T, Wu Y, Huang Y, et al: RNAi targeting CXCR4 inhibits tumor growth through inducing cell cycle arrest and apoptosis. Mol Ther 20: 398-407, 2012.

28. Coleman MP, Rachet B, Woods LM, et al: Trends and socioeconomic inequalities in cancer survival in England and Wales up to 2001. Br J Cancer 90: 1367-1373, 2004.

29. Zhang J, Cao W, Xu Q and Chen WT: The expression of EMP1 is downregulated in oral squamous cell carcinoma and possibly associated with tumour metastasis. J Clin Pathol 64: 25-29, 2010.

30. Srinivas PR, Kramer BS and Srivastava S: Trends in biomarker research for cancer detection. Lancet Oncol 2: 698-704, 2001.

31. Nishikawa M: Reactive oxygen species in tumor metastasis Cancer Lett 266: 53-59, 2008.

32. Erejuwa OO, Sulaiman SA and Ab Wahab MS: Honey: a novel antioxidant. Molecules 17: 4400-4423, 2012.

33. Tian C, Fang S, Du X and Jia C: Association of the C47T polymorphism in SOD2 with diabetes mellitus and diabetic microvascular complications: a meta-analysis. Diabetologia 54: 803-811, 2011.

34. Martin RC, Li Y, Liu Q, et al: Manganese superoxide dismutase V16A single-nucleotide polymorphism in the mitochondrial targeting sequence is associated with reduced enzymatic activity in cryopreserved human hepatocytes. DNA Cell Biol 28: 3-7, 2009.

35. Sasabe E, Yang Z, Ohno S and Yamamoto T: Reactive oxygen species produced by the knockdown of manganese-superoxide dismutase up-regulate hypoxia-inducible factor-1alpha expression in oral squamous cell carcinoma cells. Free Radic Biol Med 48: 1321-1329, 2010.

36. Shimoda-Matsubayashi S, Matsumine H, Kobayashi $\mathrm{T}$, Nakagawa-Hattori Y, Shimizu Y and Mizuno Y: Structural dimorphism in the mitochondrial targeting sequence in the human manganese superoxide dismutase gene. A predictive evidence for conformational change to influence mitochondrial transport and a study of allelic association in Parkinson's disease. Biochem Biophys Res Commun 226: 561-565, 1996.

37. Yao S, Barlow WE, Albain KS, et al: Manganese superoxide dismutase polymorphism, treatment-related toxicity and disease-free survival in SWOG 8897 clinical trial for breast cancer. Breast Cancer Res Treat 124: 433-439, 2010.

38. Xu Z, Zhu H, Luk JM, et al: Clinical significance of SOD2 and GSTP1 gene polymorphisms in Chinese patients with gastric cancer. Cancer 118: 5489-5496, 2012.

39. Kang D, Lee KM, Park SK, et al: Functional variant of manganese superoxide dismutase (SOD2 V16A) polymorphism is associated with prostate cancer risk in the prostate, lung, colorectal, and ovarian cancer study. Cancer Epidemiol Biomarkers Prev 16: 1581-1586, 2007.

40. Wheatley-Price P, Asomaning K, Reid A, et al: Myeloperoxidase and superoxide dismutase polymorphisms are associated with an increased risk of developing pancreatic adenocarcinoma. Cancer 112: 1037-1042, 2008 